\title{
Die Identität von amorphem und kristallisiertem Silicium.
}

\section{Von Wileedim Manchot.}

Meine frühere Untersuchung ${ }^{1}$ ) über die Formen des Siliciums hatte zu dem Ergebnis geführt, dab das Silicium in zwei Formen auftritt, welche in ihren chemischen Reaktionen und in ihrem Aussehen von einander sehr verschieden sind. Die eine Form ist die bekannte kristallisierte Modifikation, die andere Form ist für das blobe Auge wie auch bei ca. 1000 facher VergröBerung amorph. Letztere gewinnt man aus abgeschreckten Aluminium- oder Silberschmelzen. Sie ist äuBerst reaktionsfähig unter anderem gegen FluBsäure, und hinterläBt bei dieser Einwirkung eine noch etwas anders aussehende noch reaktionsfähigere hellbraune amorphe Form, welche mit etwas Wasserstoff beladen ist. Diese Gashülle verhindert oder verlangsamt vermutlich den Angriff der Flußsäure, während diese Form gegen andere Agentien noch heftiger reagiert.

Es entstand nun die Frage, ob das sehr deutlich verschiedene chemische Verhalten der beiden Siliciumformen zwei allotropen. Modifikationen entspricht, oder ob nur eine graduelle Verschiedenheit zwischen diesen beiden Formen besteht. Das amorphe Silicium läßt sich durch Umkristallisieren in den kristallisierten Zustand zurückverwandeln. Ich faßte damals meine Meinung in folgender Weise zusammen: „Die Gesamtheit dieser Tatsachen spricht zurzeit wohl dafür, dab es sich bei dem amorphen Silicium um ein kristallisiertes von äußerst feiner Verteilung handelt, welches eben durcb diese Verteilung eine so viel gröBere Reaktionsfähigkeit erhält, als sie das kristallisierte besitzt."

Auf meine Bitte hat nun Herr Prof. DebXe mit Herrn FradeNFELDER diese beiden amorphen Siliciumarten nach seiner Methode mit Röntgenstrahlen untersucht und gefunden, wie er mir freundlichst mitteilte, daB beide die bekannte diamantähnliche Struktur aufweisen, welche auch dem kristallisierten Silicium nach DeBre zu-

1) Ber. deutsch. chem. Ges. 54 (1921), 3107; Z. anorg. u. allg. Chem. 120. (1922), 277; 122 (1922), 22. 
kommt. ${ }^{1}$ ) Ultramikroskopische Gleichheit haben die einzelnen Teilchen noch nicht erreicht. Letzteres war mir übrigens schon durch die mikroskopische Betrachtung bei ca. 1000 facher VergröBerung bekannt. ${ }^{2}$ )

Hiermit ist die Frage entschieden. Auch in der feinsten und reaktionsfähigsten bisher dargestellten Form ist das scheinbar amorphe Silicium identisch mit dem kristallisierten und von ihm nur durch die sehr feine Verteilung verschieden. Man muB also wohl annehmen, daB durch diese feine Verteilung, wie sie durch Auflösen in Aluminium oder Silber mit nachfolgendem Abschrecken bewirkt wird, das Verhältnis zwischen Oberfäche und Masse der Substanz sich ganz enorm im Sinne einer starken Vergrößerung der ersteren verschiebt. Sonst ist die auBerordentlich viel größere Reaktionsfähigkeit der beiden pseudoamorphen Siliciumarten gegenüber der kristallisierten Art nicht $z u$ verstehen. Denn in diesem Fall kann nicht davon die Rede sein, daB durch den Angriff des lösenden Agens (Flußsäure) die Kristalloberfläche sich mit einem Überzug des Reaktionsproduktes bedecke, welcher vor weiterem Angriff schützt oder ihn verlangsamt.

Zur Charakterisierung des verschiedenen Verhaltens von pseudoamorphem, aus abgeschreckten Metallösungen gewonnenen Silicium gegenüber kristallisiertem sei der früheren Beschreibung des chemischen Verhaltens gegen Flußsäure, Alkalien, Chlor, Brom und Salpetersäure noch folgendes hinzugefügt: Zerreibt man kristallisiertes Silicium mit Bleisuperoxyd, so tritt nichts Auffallendes ein. Mit dem schwarzen durch Abschrecken seiner metallischen Lösungen gewonnenen pseudoamorphen Silicium erfolgt jedoch schon beim Zusammenreiben in der Kälte heftige Feuererscheinung und die hellbraune durch Einwirkung von FluBsäure auf das abgeschreckte Silicium gewonnene Form explodiert hierbei mit starkem Knall.

1) Physikal. Z. 17 (1916), 277.

9) Vgl. die erste Mitteilung, Z. anorg. u. allg. Chem. 120 (1922), 280.

Mïnchen, Anorganisches Laboratorium der Technischen Hochschule.

Bei der Redaktion eingegangen am 9. September 1922. 